\title{
Application of CFD Modeling Techniques to Design and Optimization of Direct-Flow Cyclones
}

\author{
$\underline{\text { Roman Havryliv }}^{1}$, Iryna Kostiv ${ }^{2}$, Volodymyr Maystruk ${ }^{3}$ \\ 1. Department of Chemical Engineering, Lviv Polytechnic National University, UKRAINE, Lviv, St.Yura square, 9, \\ E-mail: havrilivroman@gmail.com \\ 2. Department of Chemical Engineering, Lviv Polytechnic National University, UKRAINE, Lviv, St.Yura square, 9, \\ E-mail: kostiv.irina@gmail.com \\ 3. Department of design and operatin of machines, Lviv Polytechnic National University, UKRAINE, Lviv, st. \\ Profesorska, 1, \\ E-mail:vmaistruk@gmail.com
}

Abstract - In the present study, the direct-flow cyclone model with a varied set of design geometrical parameters has been analysed. Computational fluid dynamic (CFD) tools based on RANS approach and a Eulerian-Lagrangian approach were used to simulate the gas-solid flow in a cyclone separator. Keywords - direct-flow cyclone, pressure drop, separation efficiency, computational fluid dynamics, numerical modeling.

\section{Introduction}

The centrifugal separating method of dusty gases in cyclone separators has been widely used mainly for environmental protection processes in chemical engineering. This type separators of make up from 80 to $90 \%$ of the total number of dust treatment plants used in the industry and capture up about $80 \%$ of all dust emissions.

At this time, there are many different designs of cyclones. Each of cyclone separator has specific applications and is characterized by appropriate technological characteristics regarding the separation efficiency and energy consumption (pressure drop). Modern engineering research in cyclone design is aimed in two conflicting and simultaneous requirements for minimizing the pressure drop and maximizing the separation efficiency. But it is impossible to implement this approach in traditional counter-flow cyclones design.

An alternative is a transition from traditional counter-flow cyclones design to direct-flow cyclones [1,2]. This type of separators has already shown its effectiveness, that requires the continuation of scientific and engineering research.

The authors of the study suggest using computational fluid dynamics (CFD) techniques which can represent a viable tool for engineering analysis and design of cyclones. On the basis of numerical simulation and comparison with experimental results, it will obtain a reliable method for calculating gas-dynamic processes and the main parameters necessary for designing directflow cyclones.

\section{Experimental Results and Discussion}

In this study, the pressure drop, fractional separation efficiency and a flow field in directflow cyclone separator were calculated by using a CFD software Ansys Fluent 17. The modeling was performed on a desktop with Intel R CoreTM i5-3230M CPU with $2.60 \mathrm{GHz}$ and 16.00 RAM.

Turbulent gas flow inside the cyclone separator was described by using the Navier -Stokes equations of mass and momentum transport averaged by the Reynolds method (RANS) [3]. This approach is implemented in ANSYS Fluent and requires the additional input of turbulence models. Currently, there is no universal turbulence model for the simulation of all types of flows. 
Thus, the choice of an adequate model of turbulence creates a serious problem for predicting the basic technical properties of cyclones.

Since the flow in a cyclone is strongly swirled the Reynolds Renormalization Group (RNG) $\mathrm{k}-\varepsilon$ turbulence model and the Reynolds stress model (RSM) with were selected. Scalable wall functions were employed for near wall treatment. The RNG turbulence model complements with two basic equations such as the equation of kinetic energy of turbulence " $\mathrm{k}$ " and that of the rate of energy dissipation " $\varepsilon$ ". While the RNG model is a very robust and widely applicable model, it was determined to be less accurate for swirling flow due to the isotropic turbulent viscosity assumption. The RSM model is more accurate for swirling flows and is based on transport equations for all components of the Reynolds stress tensor and the respective dissipation rate. But it should be noted that the RSM model is considerably more computationally intensive [3].

Lagrangian approach with DPM (Discrete Phase Model) was used to calculate the discrete phase motion and to predict the separator efficiency in the cyclone separator. The DPM model takes into account the gravity force of the particle and the coefficient of restitution with the cyclone wall.

The analysis of the gas-solid flow numerical simulation results was performed based on the comparison with experimental data of pressure drop and separation efficiency for a different design of direct-flow cyclon separators. It was found that the differences between the values of pressure drop predicted by CFD simulations and obtained from measurements were small than $5 \%$. However, in the case of separation efficiency simulated results and experimental data were quite different for small particles diameter from 1 to $10 \mu \mathrm{m}$. This fact can be explained by accepted assumptions in used models.

\section{Conclusion}

The numerical model has been created to understand the effect of the cyclone geometrical parameters on the cyclone separation efficiency and pressure drop values. Based on experimental data and modeling results it was found that the main cause of the low pressure drop values is the direct gas-flow in the cyclone. The new cyclone design results in nearly one-half the pressure drop obtained in the traditional counter-flow cyclones design. The results obtained in this work indicate that these ideas can provide an alternative approach for new design cyclone separator creation.

\section{References}

[1] R. I. Havryliv, V. V. Maystruk, O. O. Timura, O. M. Kuhar, "Determination of hydraulic resistance of cyclones by means of computer modeling Solidworks flow simulation," Scientific herald of the National Forestry University of Ukraine, Collection of scientific works, 2013, 23, 16, pp. $364-369$.

[2] V. V. Maystruk, R. I. Havryliv, A. S. Popil, A. M. Basistyi, "Assessment of energy costs in work of direct-acting cyclone using software package flow simulation," Eastern-European Journal of Enterprise Technologies, 2012, 68, 60, pp.28- 31.

[3] B. Vandersson, R. Andersson, L. Hakansson, M. Mortensen, R. Sudiyo, B. Van Wachem, "Computational Fluid Dynamics for Engineers," Cambridge University Press, Cambridge, 2012. 